\title{
Cluster functions and scattering amplitudes for six and seven points
}

\section{Thomas Harrington and Marcus Spradlin}

Department of Physics, Brown University, Providence RI 02912, U.S.A.

E-mail: thomas_harrington@brown.edu, marcus_spradlin@brown.edu

ABSTRACT: Scattering amplitudes in planar super-Yang-Mills theory satisfy several basic physical and mathematical constraints, including physical constraints on their branch cut structure and various empirically discovered connections to the mathematics of cluster algebras. The power of the bootstrap program for amplitudes is inversely proportional to the size of the intersection between these physical and mathematical constraints: ideally we would like a list of constraints which determine scattering amplitudes uniquely. We explore this intersection quantitatively for two-loop six- and seven-point amplitudes by providing a complete taxonomy of the $\operatorname{Gr}(4,6)$ and $\operatorname{Gr}(4,7)$ cluster polylogarithm functions of [15] at weight 4 .

KeYwords: Scattering Amplitudes, Supersymmetric Gauge Theory

ARXIV EPRINT: 1512.07910 


\section{Contents}

1 Introduction 1

2 Review and notation 3

2.1 The $\operatorname{Gr}(4,6)$ cluster algebra 4

$\begin{array}{lll}2.2 & \text { The } \operatorname{Gr}(4,7) \text { cluster algebra } & 6\end{array}$

2.3 The cobracket and Bloch groups 6

3 The cluster structure of hexagon functions at weight $4 \quad 7$

3.1 Setup 7

3.2 The non-classical functions 8

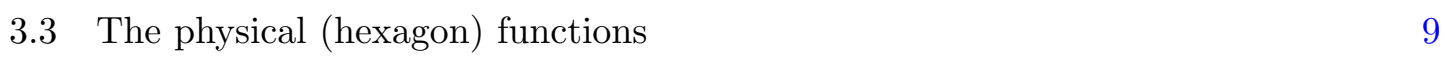

$\begin{array}{lll}3.4 \text { Summary } & 10\end{array}$

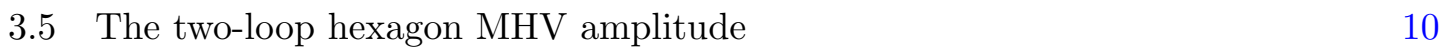

$\begin{array}{lll}3.6 & \text { The two-loop hexagon NMHV amplitude } & 11\end{array}$

4 The cluster structure of heptagon functions at weight $4 \quad 11$

$\begin{array}{lll}4.1 \text { Setup } & 11\end{array}$

$\begin{array}{ll}4.2 & \text { The non-classical functions } \\ \end{array}$

$\begin{array}{lll}4.3 & \text { The physical (heptagon) functions } & 13\end{array}$

$\begin{array}{lll}4.4 & \text { Summary } & 14\end{array}$

4.5 The two-loop heptagon MHV amplitude 14

$\begin{array}{ll}\text { 4.6 The two-loop heptagon NMHV amplitude } & 14\end{array}$

5 Conclusion $\quad 15$

$\begin{array}{ll}\text { A Two-loop heptagon NMHV coproduct data } & 16\end{array}$

$\begin{array}{lll}\text { A. } 1 f_{13} & 17\end{array}$

$\begin{array}{lll}\text { A.2 } & f_{12} & 17\end{array}$

$\begin{array}{lll}\text { A.3 } & f_{14} & 19\end{array}$

$\begin{array}{lll}\text { A.4 } & B_{1} & 20\end{array}$

\section{Introduction}

Several recent papers following [1] have explored the connection between (multi-loop) scattering amplitudes in planar $\mathcal{N}=4$ super-Yang-Mills (SYM) theory and cluster algebras, a subject of great interest to mathematicians. This line of research has two closely related branches: (1) investigating purely mathematical questions having to do with the classification of functions with certain cluster algebraic properties, i.e. "how rare are special 
functions of the type we see in SYM theory?", and (2) exploiting these mathematical properties, together with physical input as needed, to carry out calculations of new, previously intractable amplitudes, i.e. "how far can we get by exploiting the special properties of cluster algebras?".

The most basic aspect of the observed connection, supported by all evidence available to date, is that $n$-point scattering amplitudes in SYM theory have singularities only at points in $\operatorname{Conf}_{n}\left(\mathbb{P}^{3}\right)$ (the space of massless $n$-point kinematics modulo dual conformal invariance) where some cluster coordinate of the associated $\operatorname{Gr}(4, n)$ cluster algebra vanishes. More specifically, all known multi-loop amplitudes may be expressed as linear combinations of generalized polylogarithm functions written in the symbol alphabet consisting of such cluster coordinates. We expect this to be true to all loop order for all MHV and NMHV amplitudes.

Deeper connections to the underlying cluster algebra have been found for the two-loop MHV remainder functions $R_{n}^{(2)}$. The algebra of generalized polylogarithm functions modulo products admits a cobracket $\delta$ satisfying $\delta^{2}=0$, giving it the structure of a Lie coalgebra [2]. It has been observed that $\delta R_{n}^{(2)}$ has a very rigid connection to the Poisson structure on the kinematic domain $\operatorname{Conf}_{n}\left(\mathbb{P}^{3}\right)$. Specifically, the $(2,2)$ component of $\delta R_{n}^{(2)}$ can always be written as a linear combination of $\operatorname{Li}_{2}\left(-x_{i}\right) \wedge \mathrm{Li}_{2}\left(-x_{j}\right)$ for pairs of cluster coordinates having Poisson bracket $\left\{\log x_{i}, \log x_{j}\right\}=0$, while the $(3,1)$ component can always be written as a linear combination of $\operatorname{Li}_{3}\left(-x_{i}\right) \wedge \log \left(x_{j}\right)$ for pairs having $\left\{\log x_{i}, \log x_{j}\right\}= \pm 1$. These mathematical properties are tightly constraining: it has been argued in [3] that, when combined with a few physical constraints, they uniquely determine the $(2,2)$ component of $\delta R_{n}^{(2)}$ for all $n$.

It is an interesting open problem to determine whether (and, if so, precisely how) the structure of more general amplitudes may be dictated by the underlying Poisson structure on $\operatorname{Conf}_{n}\left(\mathbb{P}^{3}\right)$. This is a difficult question to address because data on multi-loop amplitudes is very hard to come by - beyond the two-loop MHV amplitudes, explicit results for complete amplitudes at fixed loop order are available only for $n=6$ [4-10] (in addition, the symbol of the two-loop $n=7$ NMHV amplitude has been computed in [11], and that of the three-loop $n=7 \mathrm{MHV}$ amplitude in [12]). With only a handful of results available it may be difficult to identify a pattern which might let one tease out the underlying structure. Moreover, accidental simplifications may occur at small $n$ which can obscure the general structure. (For example, the $(2,2)$ component of $\delta R_{6}^{(2)}$ is identically zero [13].) It is known that the $(3,3)$ component of $\delta R_{6}^{(3)}$ is not expressible in terms of cluster $\mathcal{X}$-coordinates [14], but there could be some more deeply hidden structure in this amplitude.

The primary goal of this paper is to further explore the taxonomy of two-loop cluster functions, as defined in [15], for $n=6,7$. We are particularly interested in the interplay between various mathematically natural but physically obscure conditions that certain functions can satisfy (such as the tight cluster constraints satisfied by all two-loop MHV amplitudes, mentioned above) and physically natural constraints, such as the requirement that amplitudes can only have physical branch points on the principal sheet (the so-called "first-entry condition" [16]). In previous work including [3] it has been remarked that the mathematical and physical constraints on MHV amplitudes seem almost orthogonal. One 
of our goals here is to explore this question quantitatively by fully classifying the dimensions of function spaces satisfying various properties.

We begin in section 2 with a lightning review to set some notation and terminology. In sections 3 and 4 respectively we exhaustively analyze the spaces of cluster functions on the $\operatorname{Gr}(4,6)$ and $\operatorname{Gr}(4,7)$ cluster algebras respectively of relevance to $n=6,7$-point amplitudes in planar SYM theory.

\section{Review and notation}

A kinematic configuration of $n$ massless on-shell particles, with a cyclic order (which comes naturally in gauge theories when one looks at planar scattering amplitudes), can be parameterized in terms of $n$ momentum twistors [17], $Z_{i} \in \mathbb{P}^{3}, i=1, \ldots, n$. The dual conformal symmetry of planar $n$-point amplitudes in SYM theory further implies that they are functions not on $\left(\mathbb{P}^{3}\right)^{n}$ but on the smaller space $\operatorname{Conf}_{n}\left(\mathbb{P}^{3}\right) \cong \operatorname{Gr}(4, n) /\left(\mathbb{C}^{*}\right)^{n-1}[1]$.

Viewing each $Z_{i}$ as a four-component vector of homogeneous coordinates, the Plücker coordinates are defined by $\langle i j k l\rangle \equiv \operatorname{det}\left(Z_{i} Z_{j} Z_{k} Z_{l}\right)$. Functions on $\operatorname{Conf}_{n}\left(\mathbb{P}^{3}\right)$ may be written in terms of ratios of Plücker coordinates such as

$$
\frac{\langle i j k l\rangle\langle a b c d\rangle}{\langle i j c d\rangle\langle a b k l\rangle},
$$

or more generally in terms of ratios of homogeneous polynomials in Plücker coordinates having total weight zero under rescaling any of the $Z_{i}$.

Such objects form the building blocks for the $\operatorname{Gr}(4, n)$ Grassmannian cluster algebra $[18,19]$, which is the algebra generated by certain preferred sets of coordinates on $\operatorname{Gr}(4, n)$. These coordinates come in two related varieties: the $\mathcal{A}$-coordinates, which consist of the Plücker coordinates and certain homogeneous polynomials in them, and the $\mathcal{X}$-coordinates [20], which consist of certain scale-invariant ratios of $\mathcal{A}$-coordinates.

In this paper we focus on the cases $n=6,7$, for which the corresponding cluster algebras have respectively $15,49 \mathcal{A}$-coordinates and $15,385 \mathcal{X}$-coordinates. ${ }^{1}$ The reader may find these coordinates tabulated in [1]. Of course, the $\mathcal{X}$-coordinates are not algebraically independent since the dimension of $\operatorname{Conf}_{n}\left(\mathbb{P}^{3}\right)$ is only $3(n-5)$. A "cluster" is a particular choice of $3(n-5)$ cluster $\mathcal{X}$-coordinates in terms of which all others may be determined by a simple set of rational transformations called mutations.

A still mysterious but apparently important role is played by the fact that $\operatorname{Conf}_{n}\left(\mathbb{P}^{3}\right)$ admits a natural Poisson structure, which it inherits from the Grassmannian [18]. A characteristic feature of cluster coordinates is that within each cluster, the $\mathcal{X}$-coordinates are log-canonical with respect to this Poisson structure, i.e.

$$
\left\{\log x_{i}, \log x_{j}\right\}=B_{i j}, \quad i, j=1, \ldots, 3(n-5),
$$

where $B$ is an antisymmetric integer-valued matrix (which for $n=6,7$ only takes the values $0, \pm 1)$.

\footnotetext{
${ }^{1}$ In some applications it is sensible to count $x$ and $1 / x$ separately, in which case these numbers would be 30,770 .
} 
We expect all six- and seven-point $L$-loop scattering amplitudes in planar SYM theory to be (generalized) polylogarithm functions of uniform transcendental weight $2 L$ whose symbols may be written in terms of the $\operatorname{Gr}(4, n)$ cluster coordinates. For the purpose of writing a symbol alphabet the relevant question is not how many coordinates are algebraically independent, but how many are multiplicatively independent - we say that a finite collection $\left\{y_{1}, \ldots, y_{m}\right\}$ is multiplicatively independent if there is no collection of integers $\left\{n_{1}, \ldots, n_{m}\right\}$ such that $\prod y_{i}^{n_{i}}=1$, i.e. if the collection $\left\{\log y_{1}, \ldots, \log y_{m}\right\}$ is linearly independent over $\mathbb{Z}$.

As mentioned above there are respectively 15 (385) cluster $\mathcal{X}$-coordinates $x_{i}$ for $n=6$ $(n=7)$, but the corresponding sets of $\log x_{i}$ only span spaces of dimension 9 (42). Choosing bases for these spaces provides a collection of 9 (42) multiplicatively independent ratios to serve as symbol alphabets for building cluster polylogarithm functions.

\subsection{The $\operatorname{Gr}(4,6)$ cluster algebra}

For six-point amplitudes the relevant cluster algebra is $\operatorname{Gr}(4,6)$, which is isomorphic to the $A_{3}$ cluster algebra. Its 15 cluster $\mathcal{A}$-coordinates are just the Plücker coordinates $\langle i j k l\rangle$. This algebra has $15 \mathcal{X}$-coordinates. In the notation of [15] these are named $v_{i}, x_{i}^{ \pm}$for $i=1,2,3$ and $e_{i}$ for $i=1, \ldots, 6$.

The reader may find explicit formulas for these as ratios of Plücker coordinates in [15]. Since one of the goals of this paper is to make contact with the work of Dixon et al. we will instead provide this information via the connection to the variables $u, v, w, y_{u}, y_{v}, y_{w}$ used in [4-10].

The three-dimensional kinematic configuration space $\operatorname{Conf}_{6}\left(\mathbb{P}^{3}\right)$ may be parameterized in terms of the three coordinates

$$
y_{u}=\frac{\langle 1236\rangle\langle 1345\rangle\langle 2456\rangle}{\langle 1235\rangle\langle 1246\rangle\langle 3456\rangle}, \quad y_{v}=\frac{\langle 1235\rangle\langle 1456\rangle\langle 2346\rangle}{\langle 1234\rangle\langle 1356\rangle\langle 2456\rangle}, \quad y_{w}=\frac{\langle 1246\rangle\langle 1356\rangle\langle 2345\rangle}{\langle 1256\rangle\langle 1345\rangle\langle 2346\rangle} .
$$

Note that a cyclic rotation $Z_{i} \rightarrow Z_{i+1}$ maps

$$
y_{u} \rightarrow 1 / y_{v}, \quad y_{v} \rightarrow 1 / y_{w}, \quad y_{w} \rightarrow 1 / y_{u},
$$

while reflection $Z_{i} \rightarrow Z_{1-i}$ (all indices are understood to be cyclic modulo 6 ) takes

$$
y_{u} \rightarrow y_{v}, \quad y_{v} \rightarrow y_{u}, \quad y_{w} \rightarrow y_{w}
$$

The spacetime parity operator acts on momentum twistors as ${ }^{2}$

$$
Z_{i} \rightarrow W_{i}=*\left(Z_{i-1} \wedge Z_{i} \wedge Z_{i+1}\right)
$$

which transforms the cross-ratios defined in (2.3) according to

$$
y_{u} \rightarrow 1 / y_{u}, \quad y_{v} \rightarrow 1 / y_{v}, \quad y_{w} \rightarrow 1 / y_{w} .
$$

\footnotetext{
${ }^{2}$ The notation means that $W_{i}$ spans the one-dimensional subspace orthogonal to the 3-plane spanned by $Z_{i-1}, Z_{i}, Z_{i+1}$ in $\mathbb{C}^{4}$.
} 
It is a curious accident that for $n=6$ spacetime parity reversal is equivalent on $\operatorname{Conf}_{n}\left(\mathbb{P}^{3}\right)$ to an element (namely, shift-by-three) of the cyclic group.

Three other variables used by Dixon et al. may be defined in terms of these via

$$
u=\frac{y_{u}\left(1-y_{v}\right)\left(1-y_{w}\right)}{\left(1-y_{u} y_{v}\right)\left(1-y_{u} y_{w}\right)}, \quad v=\frac{y_{v}\left(1-y_{u}\right)\left(1-y_{w}\right)}{\left(1-y_{u} y_{v}\right)\left(1-y_{v} y_{w}\right)}, \quad w=\frac{y_{w}\left(1-y_{u}\right)\left(1-y_{v}\right)}{\left(1-y_{u} y_{w}\right)\left(1-y_{v} y_{w}\right)} .
$$

Central to our investigations is the Poisson structure on $\operatorname{Conf}_{6}\left(\mathbb{P}^{3}\right)$, which may be expressed in terms of the $y$ variables as

$$
\left\{\log y_{u}, \log y_{v}\right\}=\left\{\log y_{v}, \log y_{w}\right\}=\left\{\log y_{w}, \log y_{u}\right\}=\frac{\left(1-y_{u}\right)\left(1-y_{v}\right)\left(1-y_{w}\right)}{1-y_{u} y_{v} y_{w}} .
$$

It is invariant under the full cyclic group (and hence, it is parity symmetric) but antisymmetric under reflection.

In terms of these variables, the cluster $\mathcal{X}$-coordinates may be expressed as

$$
\begin{aligned}
& v_{1}=\frac{1-v}{v}, \quad v_{2}=\frac{1-w}{w}, \quad v_{3}=\frac{1-u}{u}, \\
& x_{1}^{+}=\frac{y_{v}\left(1-y_{u} y_{w}\right)}{1-y_{v}}, \quad x_{2}^{+}=\frac{y_{w}\left(1-y_{u} y_{v}\right)}{1-y_{w}}, \quad x_{3}^{+}=\frac{y_{u}\left(1-y_{v} y_{w}\right)}{1-y_{u}}, \\
& x_{1}^{-}=\frac{1-y_{u} y_{w}}{y_{u} y_{w}\left(1-y_{v}\right)}, \quad x_{2}^{-}=\frac{1-y_{u} y_{v}}{y_{u} y_{v}\left(1-y_{w}\right)}, \quad x_{3}^{-}=\frac{1-y_{v} y_{w}}{y_{v} y_{w}\left(1-y_{u}\right)}, \\
& e_{1}=\frac{1-y_{v}}{y_{v}\left(1-y_{u}\right)}, \quad e_{2}=\frac{y_{v}\left(1-y_{w}\right)}{1-y_{v}}, \quad e_{3}=\frac{1-y_{u}}{y_{u}\left(1-y_{w}\right)}, \\
& e_{4}=\frac{y_{u}\left(1-y_{v}\right)}{1-y_{u}}, \quad e_{5}=\frac{1-y_{w}}{y_{w}\left(1-y_{v}\right)}, \quad e_{6}=\frac{y_{w}\left(1-y_{u}\right)}{1-y_{w}} \text {. }
\end{aligned}
$$

Note that under a cyclic shift $Z_{i} \rightarrow Z_{i+1}$ we have

$$
v_{i} \rightarrow v_{i+1}, \quad x_{i}^{ \pm} \rightarrow x_{i+1}^{\mp}, \quad e_{i} \rightarrow e_{i+1},
$$

while under parity the $v_{i}$ are invariant and

$$
x_{i}^{ \pm} \rightarrow x_{i}^{\mp}, \quad e_{i} \rightarrow e_{i+3} .
$$

Of particular importance are pairs $x_{1}, x_{2}$ of distinct $\mathcal{X}$-coordinates with simple Poisson brackets. By "simple" we mean specifically that $\left\{\log x_{1}, \log x_{2}\right\}$ is either 0 or \pm 1 . There are three pairs with Poisson bracket zero,

$$
\left\{\log x_{i}^{+}, \log x_{i}^{-}\right\}=0
$$

and 30 pairs with Poisson bracket +1 ,

$$
\left\{\log e_{i}, \log e_{i+4}\right\}=\left\{\log x_{i+1}^{ \pm}, \log v_{i}\right\}=\left\{\log v_{i+1}, \log x_{i}^{ \pm}\right\}=\left\{\log x_{i+1}^{ \pm}, \log e_{i}\right\}=1
$$

together with their cyclic images, for $6+6+6+12=30$ pairs. The remaining 72 pairs have "complicated" Poisson brackets (specifically, non-integer-valued; see for example (2.9)). 


\subsection{The $\operatorname{Gr}(4,7)$ cluster algebra}

For seven-point amplitudes the relevant cluster is algebra is $\operatorname{Gr}(4,7)$, which is isomorphic to the $E_{6}$ algebra. The 49 cluster $\mathcal{A}$-coordinates consist of the 35 Plücker coordinates $\langle i j k l\rangle$ together with 14 homogeneous polynomials denoted by $\langle 1(23)(45)(67)\rangle,\langle 2(13)(45)(67)\rangle$ (and their cyclic images), where

$$
\langle i(i-1, i+1)(j, j+1)(k, k+1)\rangle=\langle i-1 i j j+1\rangle\langle i i+1 k k+1\rangle-\langle i-1 i k k+1\rangle\langle i i+1 j j+1\rangle .
$$

One can build from these $49 \mathcal{A}$-coordinates a total of 385 cluster $\mathcal{X}$-coordinates (or 770 if we count their multiplicative inverses). These are tabulated on pages 40-41 of [1]. Out of $\frac{1}{2} \cdot 385 \cdot 384=73920$ pairs of $\mathcal{X}$-coordinates, 2520 have Poisson bracket \pm 1 while 833 have Poisson bracket zero.

\subsection{The cobracket and Bloch groups}

We recall that the algebra $\mathcal{A}$ of generalized polylogarithm functions admits a coproduct giving it the structure of a Hopf algebra [2]. When we work with the quotient space $\mathcal{L}$ of polylogarithm functions modulo products of functions of lower weight, the coproduct descends onto the quotient space to a cobracket $\delta$ which satisfies $\delta^{2}=0$. We review here only the barest essentials, and refer the reader to $[1,15]$ for additional details.

The cobracket of a weight-4 function has two components,

$$
\delta \mathcal{L}_{4} \in\left(B_{3} \otimes \mathbb{C}^{*}\right) \oplus\left(B_{2} \wedge B_{2}\right),
$$

where the Bloch group $B_{k}$ is, for our purposes, the free abelian group generated by functions of the form $\{x\}_{k} \equiv-\operatorname{Li}_{k}(-x)$, where $\mathrm{Li}_{k}$ is the classical polylogarithm function and $x$ is a function on $\operatorname{Conf}_{n}\left(\mathbb{P}^{3}\right)$ which is rational in Plücker coordinates.

The fact that $\delta^{2}=0$ and that $\delta$ has trivial cohomology means that if $a \in B_{3} \otimes \mathbb{C}^{*}$ and $b \in B_{2} \wedge B_{2}$, then there exists a function $f$ whose cobracket components are $a \oplus b$ if and only if $\delta_{31}(a)+\delta_{22}(b)=0$. As explained in [15], this condition can be used to explicitly enumerate cluster functions, at least on algebras of finite type. For such algebras $B_{3} \otimes \mathbb{C}^{*}$ and $B_{2} \wedge B_{2}$ are finite dimensional vector spaces on which $\delta$ acts linearly, so the space of cluster $\mathcal{A}$-functions is simply the kernel of $\delta$.

At weight 4 a general polylogarithm can be expressed in terms of the classical functions $\mathrm{Li}_{k}$ if and only if its $B_{2} \wedge B_{2}$ cobracket component vanishes. We will often be interested in counting the number of non-classical functions, since the classical ones (which correspond to solutions of $\left.\delta_{31}(a)=0\right)$ are trivial to enumerate. To answer this question we compute the dimension of the subspace of $B_{2} \wedge B_{2}$ such that the equation $\delta_{31}(a)+\delta_{22}(b)=0$ is solvable for some $a \in B_{3} \otimes \mathbb{C}^{*}$.

One final piece of terminology concerns the interplay between the Poisson structure on the Grassmannian cluster algebras and the cobracket of polylogarithm functions. We recall that two cluster $\mathcal{X}$-coordinates $x, y$ have $\{\log x, \log y\} \in \mathbb{Z}$ only if there exists a cluster containing either $x$ or $1 / x$, and either $y$ or $1 / y$. As reviewed in [1], the combinatorics of mutations is encoded in a graph called the (generalized) Stasheff polytope associated to the 
algebra. We therefore say that a function has "Stasheff local" $B_{2} \wedge B_{2}$ if it can be expressed as a linear combination of terms of the form $\{x\}_{2} \wedge\{y\}_{2}$ for pairs having integer Poisson bracket (for $\operatorname{Gr}(4,6)$ and $\operatorname{Gr}(4,7)$, this integer will always be in the set $\{-1,0,+1\}$ ).

\section{The cluster structure of hexagon functions at weight 4}

\subsection{Setup}

In this section we consider cluster functions on the $A_{3} \cong \operatorname{Gr}(4,6)$ cluster algebra. The term "cluster $\mathcal{A}$-function" introduced in [15] refers, in the present application, to an integrable symbol written in the 9-letter alphabet of cluster coordinates (specifically, this means any multiplicatively independent set of $\mathcal{X}$-coordinates; or equivalently, homogeneous ratios of $\mathcal{A}$-coordinates) on $\operatorname{Gr}(4,6)$.

Any linear combination of cluster $\mathcal{A}$-functions with the property that only the three variables $u, v, w$ appear in the first-entry of the symbol, reflecting the physically allowed branch points for a scattering amplitude [16], is called a "physical function" or, following the terminology of [6], a "hexagon function". These have been studied through high weight in the series of papers [4-10], but we restrict our analysis to weight 4 as our aim is to explore connections between the cobrackets and the cluster Poisson structure of these functions.

Let $\mathcal{A}_{k}$ denote the vector space of all weight- $k$ cluster $\mathcal{A}$-functions. Such functions are easy to count for any $A_{m}$ type cluster algebra (see [21, 22]); for $A_{3}$ we have the generating function

$$
f_{A_{3}}(t)=1+\sum_{k=1}^{\infty} t^{k} \operatorname{dim}\left(\mathcal{A}_{k}\right)=\frac{1}{1-2 t} \frac{1}{1-3 t} \frac{1}{1-4 t}
$$

so that

$$
\operatorname{dim}\left(\mathcal{A}_{k}\right)=9,55,285,1351, \ldots \quad k=1,2,3,4, \ldots
$$

Let $\mathcal{L}_{k}$ denote the quotient of $\mathcal{A}_{k}$ by products of functions of lower weight. The number of such functions can be computed by taking the plethystic logarithm of the generating function $f_{A_{3}}(t)$ (see for example [23]), which gives

$$
\operatorname{dim}\left(\mathcal{L}_{k}\right)=9,10,30,81, \ldots \quad k=1,2,3,4, \ldots
$$

Finally we denote by $B_{k}$ the subspace of $\mathcal{L}_{k}$ generated by the classical polylogarithms (we do not yet restrict their arguments to be cluster $\mathcal{X}$-coordinates). We have

$$
\operatorname{dim}\left(\mathcal{B}_{k}\right)=10,30,45, \ldots \quad k=2,3,4, \ldots
$$

For $k<4$ the agreement with (3.3) reflects the fact that all such generalized polylogarithms can be expressed in terms of the classical functions; for higher $k$ these numbers can be obtained by choosing a basis for $\mathcal{L}_{k}$ and computing dimker $\delta$ as described in the previous section. 


\subsection{The non-classical functions}

Beginning at $k=4$ we can distinguish between classical and non-classical functions. At weight $k=4$, the "non-classicalness" of a function is completely characterized by its $B_{2} \wedge B_{2}$ cobracket component (see for example [1]). Since $B_{2}$ has dimension 10 according to (3.4), $B_{2} \wedge B_{2}$ evidently has dimension 45 . However, a random element of this vector space is not guaranteed to be the $B_{2} \wedge B_{2}$ cobracket component of any cluster $\mathcal{A}$-function - there is a nontrivial integrability constraint.

In fact, by comparing (3.4) to (3.3) we see that there are 81 functions in all, minus 45 classical functions, for a total of 36 non-classical functions. We conclude that in the 45-dimensional space $B_{2} \wedge B_{2}$ spanned by objects of the form $\{x\}_{2} \wedge\{y\}_{2}$, for cluster coordinates $x$ and $y$, only the linear combinations lying in a particular 36-dimensional subspace correspond to cobracket components of actual cluster $\mathcal{A}$-functions. ${ }^{3}$ We will shortly characterize this 36-dimensional space completely.

Let us write $P B_{0}$ to denote the subspace of $B_{2} \wedge B_{2}$ spanned by objects of the form $\{x\}_{2} \wedge\{y\}_{2}$ for pairs having Poisson bracket $\{\log x, \log y\}=0$. In what follows we will for example say that a function "lives in $P B_{0}$ " if its $B_{2} \wedge B_{2}$ cobracket component can be expressed in terms of such pairs. Similarly, let $P B_{1}$ be the subspace spanned by pairs having Poisson bracket 1 , and let us also use the shorthand $P B_{*}=B_{2} \wedge B_{2}$, meaning that the Poisson bracket can be anything. We found in (2.13) and (2.14) that there are respectively 3, 30 pairs with Poisson bracket 0,1 . It is simple to check that the corresponding elements are linearly independent in $B_{2} \wedge B_{2}$, so we have that $\operatorname{dim} P B_{0}=3$ and $\operatorname{dim} P B_{1}=30$, while of course $\operatorname{dim} P B_{*}=\operatorname{dim} B_{2} \wedge B_{2}=45$.

With this notation in hand let us now summarize our findings on the 36 non-classical cluster $\mathcal{A}$-functions at weight four, which we find fall into two broad groups:

(A) 6 of these functions are the " $A_{2}$ cluster functions" introduced in [15]. There is one such function for each $A_{2}$ subalgebra of $A_{3}$; these subalgebras and the associated functions are represented visually in equation (4.3) of that paper. These six functions have additional "cluster structure": their $B_{3} \otimes \mathbb{C}^{*}$ cobracket components can be expressed entirely in terms of cluster $\mathcal{X}$-coordinates - this means that they are "cluster $\mathcal{X}$-functions" in the terminology of [15]. General elements of this six-dimensional space are not Stasheff local - their $B_{2} \wedge B_{2}$ cobracket components are not expressible in terms of pairs of coordinates with Poisson bracket $0, \pm 1$. Only one particular linear combination of these 6 - the one called the $A_{3}$ function in [15] — has a nice $B_{2} \wedge B_{2}$, in fact lying inside $P B_{0}$. The $B_{2} \wedge B_{2}$ cobracket component of this $A_{3}$ function is

$$
\sum_{i=1}^{3}\left\{x_{i}^{+}\right\}_{2} \wedge\left\{x_{i}^{-}\right\}_{2} .
$$

This quantity is parity-odd so it cannot possibly appear in the two-loop six-point MHV remainder function, which is parity-even. This "explains" why the hypothesis that two-

\footnotetext{
${ }^{3}$ Linear combinations which fall outside this 36-dimensional subspace are certainly integrable [24], but they integrate to functions with symbols involving letters which are not cluster coordinates, for example differences of $\mathcal{X}$-coordinates $x_{i}-x_{j}$, which does not in general factor into a product of cluster coordinates. Hence they are not cluster $\mathcal{A}$-functions.
} 
loop MHV remainder functions must live in $P B_{0}$, which we know to be true for all $n$ [3], implies that the case $n=6$ must be classical.

(B) The remaining 30 functions are sort of the opposite: no linear combination of these 30 has a $B_{3} \otimes \mathbb{C}^{*}$ content which can be expressed entirely in terms of $\mathcal{X}$-coordinates, so none of them are cluster $\mathcal{X}$-functions. On the other hand, all of them are Stasheff local - they all have "nice" $B_{2} \wedge B_{2}$, in fact they span exactly the 30 -dimensional subspace $P B_{1} \subset B_{2} \wedge B_{2}$.

\subsection{The physical (hexagon) functions}

Dixon et al. find that there are precisely 15 functions at weight 4 (modulo products of functions of lower weight) satisfying the first-entry condition, which they call hexagon functions. Let us put aside 9 which are purely classical and focus on the two types of functions named $\Omega_{2}$ and $F_{1}$ in [6].

(A) The function $F_{1}$ is parity-odd and comes in three cyclic permutations (i.e., $i \rightarrow i+2$ and $i \rightarrow i+4)$. These functions are rather interesting; each of them has a $B_{2} \wedge B_{2}$ coproduct component given by (3.5) plus additional terms which cannot be expressed in terms of pairs having simple Poisson bracket. Since (3.5) is invariant under $i \rightarrow i+2$, we can throw out these terms by taking the difference between any two pairs of the three permutations of $F_{1}$. Indeed such linear combinations have appeared in the literature, as in (B.18) and (B.20) of [6] which define the function $\widetilde{V}$ by

$$
8 \widetilde{V}=-F_{1}(u, v, w)+F_{1}(w, u, v)+\text { products of lower-weight functions. }
$$

Hence only two of the three distinct cyclic permutations of $\widetilde{V}$ are linearly independent.

(B) Next we look at the parity-even function $\Omega_{2}$ which also comes in three cyclic permutations. At the level of $B_{2} \wedge B_{2}$, where we can ignore all terms involving only classical polylogarithms, the function $\Omega_{2}$ is equivalent (modulo an overall multiplicative factor) to the function called $V$ by Dixon et al.; see for example (7.1) through (7.3) of [4]. In that paper it was also observed that the three cyclic permutations of this function add up to a purely classical function, so the three different permutations of $V$ span only a two-dimensional subset of $B_{2} \wedge B_{2}$.

To summarize, we find that the subspace of $B_{2} \wedge B_{2}$ spanned by physical (hexagon) functions has dimension 5. Two dimensions are spanned by the parity-even functions of type $V$, while three dimensions are spanned by the parity-odd functions of type $F_{1}$. Although a generic vector in the three-dimensional parity-odd subspace has terms with "bad" Poisson brackets, there is something especially nice about the subspace spanned by the permutations of $V$ and $\widetilde{V}$ together. To see this we exhibit here a formula for their cobracket components, which we find are most simply packaged in the formula

$$
\left.\delta\right|_{2,2}(V+\widetilde{V})=\frac{1}{2}\left\{v_{2}\right\}_{2} \wedge\left\{x_{1}^{-}\right\}_{2}-\frac{1}{2}\left\{v_{1}\right\}_{2} \wedge\left\{x_{3}^{-}\right\}_{2}-\frac{1}{2}\left\{x_{1}^{+}\right\}_{2} \wedge\left\{v_{3}\right\}_{2}+\frac{1}{2}\left\{x_{2}^{+}\right\}_{2} \wedge\left\{v_{1}\right\}_{2} .
$$

Since $V, \widetilde{V}$ have parity even and odd, respectively, $\left.\delta\right|_{2,2}(V-\widetilde{V})$ is given by the same formula but with $x^{ \pm} \rightarrow x^{\mp}$. We now see that each term in (3.7) involves only the $P B_{1}$ 
pairs listed in (2.14)! Moreover, it is trivial to check directly from (3.7) and the cyclic transformations (2.11) that the six functions $V, \widetilde{V}$ altogether span only a four-dimensional subspace of $P B_{1}$.

\subsection{Summary}

The results of this section can be summarized in the following classification of weight- 4 cluster functions on $A_{3} \cong \operatorname{Gr}(4,6)$ :

There are a total of 81 irreducible weight-four cluster $\mathcal{A}$-functions

$\llcorner 45$ classical, 10 of which are physical

$\llcorner 36$ non-classical, 5 of which are physical

(three permutations of $F_{1}$ and two of $\Omega_{2}$ )

$\iota 30 P B_{1}$ functions, 4 of which are physical

(two permutations each of $V, \tilde{V}$ )

$\left\llcorner 6 A_{2}\right.$ functions; these are all of the cluster $\mathcal{X}$-functions

$\iota 1 P B_{0}$ function, the $A_{3}$ function

(as mentioned above, this is $F_{1}$ plus terms in $P B_{*}$ )

$\left\llcorner 5 P B_{*}\right.$ functions

Let us emphasize that these numbers count only irreducible functions, and that starting from the third line they moreover count functions modulo the classical function $\mathrm{Li}_{4}$ (i.e., the numbers refer to dimensions of subspaces of $B_{2} \wedge B_{2}$ ). When we say that a function is physical modulo additional terms, we mean that it is possible to choose the additional terms to render the function physical.

\subsection{The two-loop hexagon MHV amplitude}

Let us now comment on the relevance of these functions to the two-loop six-point MHV remainder function $R_{6}^{(2)}$, which was found to be expressible in terms of the classical polylogarithm functions $\mathrm{Li}_{k}$ in [13] (a fact that we "explained" below (3.5)). In fact, this amplitude is even more special because it is a cluster $\mathcal{X}$-function, which means that it can be expressed in entirely in terms of the $\operatorname{Li}_{k}(-x)$; the $\operatorname{Li}_{k}(1+x)$ and $\operatorname{Li}_{k}(1+1 / x)$ functions, whose $B_{3} \otimes \mathbb{C}^{*}$ cobracket components are not expressible in terms of cluster $\mathcal{X}$-coordinates, are not needed [1].

Above we tabulated our finding that (modulo products of lower-weight functions) there are only 10 physical and classical polylogarithms at weight four. In this space we now search for functions whose coproducts are expressible entirely in terms of the $\operatorname{Li}_{k}(-x)$. We find that there is a unique linear combination that is invariant under the discrete symmetries (parity and dihedral invariance) that MHV amplitudes must possess. That linear combination is proportional to the two-loop MHV remainder function

$R_{6}^{(2) \mathrm{MHV}}=\sum_{i=1}^{3}\left[\operatorname{Li}_{4}\left(-x_{i}^{+}\right)+\operatorname{Li}_{4}\left(-x_{i}^{-}\right)-\frac{1}{2} \operatorname{Li}_{4}\left(-v_{i}\right)\right]+$ products of lower-weight functions 
in agreement with the known result [13]. (This argument, of course, does not fix the overall coefficient.) Of course, in this case it is very well known that the product terms are also completely fixed by simple considerations, but our focus in this paper is on the leading term.

\subsection{The two-loop hexagon NMHV amplitude}

The $n=6$ NMHV two-loop ratio function is given by [4]

$$
\mathcal{P}_{6, \mathrm{NMHV}}^{(2)}=[23456]\left[V(u, v, w)+\widetilde{V}\left(y_{u}, y_{v}, y_{w}\right)\right]+\text { cyclic }
$$

where [23456] is the $R$-invariant

$$
[a b c d e]=\frac{\delta^{4}\left(\chi_{a}\langle b c d e\rangle+\text { cyclic }\right)}{\langle a b c d\rangle\langle b c d e\rangle\langle c d e a\rangle\langle d e a b\rangle\langle e a b c\rangle}
$$

and $V, \widetilde{V}$ are the two generalized polylogarithm functions of uniform transcendental weight four reviewed in section 3.3 above. These two functions were computed explicitly in [4] (see also [21] for a different presentation of these functions). The $B_{2} \wedge B_{2}$ component of the cobracket of this amplitude was computed in (3.7), where it was found to be expressible entirely in terms of pairs living in $P B_{1}{ }^{4}$

The NMHV ratio function provides us (at the level of $B_{2} \wedge B_{2}$ ) with a total of four linearly independent non-classical functions of weight 4 (as reviewed above, each of $V$ and $\widetilde{V}$ comes in three cyclic permutations, but the cyclic sum of each is separately zero inside $B_{2} \wedge B_{2}$ ). We see from the summary in section 3.4 that precisely 5 functions of this type exist. Only four linear combinations of them, however, actually appear in the amplitude - these are precisely the four linear combinations which live in $P B_{1}$ ! The one additional non-classical weight-4 hexagon function which exists but does not appear in the amplitude, $F_{1}$ by itself, has terms with "bad" Poisson brackets (i.e., non-Stasheff local terms) in its $B_{2} \wedge B_{2}$ content.

\section{The cluster structure of heptagon functions at weight 4}

\subsection{Setup}

In this section the term "cluster function" refers to an integrable symbol written in the 42 letter alphabet of cluster coordinates on $\operatorname{Gr}(4,7)$. Any linear combination of such symbols with the property that only the Plücker coordinates of the form $\langle i i+1 j j+1\rangle$ appear in the first entry of the symbol, reflecting the physically allowed branch points for a scattering amplitude, is called (the symbol of) a "physical function" or a "heptagon function" following the terminology of [12] where they have been studied through weight six. The analysis here, where we aim to make finer statements about the connection to the Poisson bracket of the cluster algebra, is again restricted to weight 4, of relevance to two-loop amplitudes.

Let $\mathcal{A}_{k}$ denote the vector space of all weight- $k$ functions. In contrast to the $A_{m}$ cluster algebras and the example shown in (3.1), we do not know of any generating function which

\footnotetext{
${ }^{4}$ This observation was first made by C. Vergu [14].
} 
counts the number of cluster functions for the $E_{6}$ algebra. These may be tabulated through weight 3 by explicit enumeration, but at higher weight these numbers must be computed by analyzing the integrability constraint. This boils down to a linear algebra problem, since counting the number of cluster functions at weight $k$ is the same as finding how many linear combinations of the $42^{k}$ weight- $k$ symbols satisfy the integrability constraint. (This calculation can be rendered more manageable by imposing integrability at the level of the cobracket rather than at the level of the symbol.) We have carried this out at $k=4$ to find that

$$
\operatorname{dim}\left(\mathcal{A}_{k}\right)=42,1035,19536,312578, \ldots \quad k=1,2,3,4, \ldots
$$

Let $\mathcal{L}_{k}$ denote the quotient of $\mathcal{A}_{k}$ by products of functions of lower weight. As in (3.3) taking the plethystic logarithm [23] gives

$$
\operatorname{dim}\left(\mathcal{L}_{k}\right)=42,132,748,4193, \ldots \quad k=1,2,3,4, \ldots
$$

Finally we denote by $B_{k}$ the subspace of $\mathcal{L}_{k}$ generated by the classical polylogarithms (we do not yet restrict their arguments to be cluster $\mathcal{X}$-coordinates). We have

$$
\operatorname{dim}\left(\mathcal{B}_{k}\right)=132,748,1155, \ldots \quad k=2,3,4, \ldots
$$

As mentioned before, agreement of these numbers with (4.2) is guaranteed for $k<4$, and we obtained the value 1155 for $k=4$ by computing $\operatorname{dim} \operatorname{ker} \delta$ as described in section 2 .

Before we turn to weight 4 , a minor interesting comment about $k=3$ is in order. It is simple to write down classical cluster functions of the form $\operatorname{Li}_{k}(-x), \operatorname{Li}_{k}(1+x)$ and $\operatorname{Li}_{k}(1+1 / x)$ for any weight $k$, where $x$ runs over the set of $385 \mathcal{X}$-coordinates. For $k=3$, this set of functions is overcomplete due to the identity

$$
\mathrm{Li}_{3}(-x)+\mathrm{Li}_{3}(1+x)+\mathrm{Li}_{3}(1+1 / x)=0 \text { mod products of lower-weight functions. (4.4) }
$$

Among the 385 functions of type $\operatorname{Li}_{3}(-x)$ there are exactly 22 additional linear relations. These were discovered in [1], where they were called $D_{4}$ identities since the simplest manifestation of this identity occurs for the $D_{4}$ algebra. Altogether then these identities account for the $3 \times 385-385-22=748$ linearly independent weight- 3 cluster $\mathcal{A}$-functions tabulated in (4.2).

\subsection{The non-classical functions}

Let us now repeat the analysis done in the beginning of section 3.2 for the $E_{6}$ algebra. Since $B_{2}$ has dimension 132, $B_{2} \wedge B_{2}$ has dimension 8646. We again use the notation $P B_{0}, P B_{1}$, and $P B_{*}=B_{2} \wedge B_{2}$ to denote the subspaces spanned by elements of the form $\{x\}_{2} \wedge\{y\}_{2}$ for pairs $x, y$ having Poisson bracket $0, \pm 1$, or "anything." We find that $P B_{0}$ has dimension 455 and $P B_{1}$ has dimension 2520.

A quick glance at (4.2) and (4.3) reveals that there are 4193-1155 $=3038$ non-classical cluster functions at weight $k=4$. We find that these fall into three groups:

(A) First, there are the $A_{2}$ functions. We recall from (for example) [1] that $E_{6}$ has 1071 $A_{2}$ subalgebras, so one can construct $1071 A_{2}$ functions according to the definition 
given in [15], but only 448 of these are linearly independent inside $B_{2} \wedge B_{2} .{ }^{5}$ These functions are moreover cluster $\mathcal{X}$-functions: their $B_{3} \otimes \mathbb{C}^{*}$ cobracket components can be expressed entirely in terms of cluster $\mathcal{X}$-coordinates, but their $B_{2} \wedge B_{2}$ content is, in general, not Stasheff local - not expressible in terms of pairs with Poisson bracket $0, \pm 1$.

There are no linear combinations of these 448 functions which live in $P B_{1}$ - these are covered in (B) just ahead - but we find that 195 linear combinations live in $P B_{0}$. This 195-dimensional space is spanned by the set of $A_{3}$ functions associated to the various $A_{3}$ subalgebras of $E_{6}$.

(B) There are 2520 functions which span the 2520-dimensional subspace $P B_{1} \subset B_{2} \wedge B_{2}$. We found the same phenomenon in the six-point case discussed in the previous section. There we furthermore found that no linear combination of these $P B_{1}$ functions had a $B_{3} \otimes \mathbb{C}^{*}$ component that could be expressed entirely in terms of $\mathcal{X}$-coordinates. We have not repeated this analysis for the 2520 seven-point functions; the computation seems formidable.

(C) There are an additional $3038-448-2520=70$ functions which we can tabulate explicitly (at least at the level of their cobrackets), but seem to have no nice characterization.

\subsection{The physical (heptagon) functions}

It was found in [12] that there are precisely 1288 functions at weight 4 satisfying the firstentry condition, which are called physical, or heptagon functions. We have computed the $B_{2} \wedge B_{2}$ cobracket of each of them, and found that there are only 126 non-zero linear combinations. This means that there are 1162 classical heptagon functions and 126 nonclassical heptagon functions at weight 4 . We have found that these 126 heptagon functions fall into three types:

(A) A total of 105 of these functions live in $P B_{0}$; they come in 15 families related by cyclic permutations.

(B) A total of 14 of these functions live in $P B_{1}$; they come in 2 families related by cyclic permutations.

(C) There is one remaining family of 7 functions related by cyclic permutations. No linear combination of these is Stasheff local (i.e., lives within the union of $P B_{0}$ and $P B_{1}$ ).

\footnotetext{
${ }^{5}$ This result was first obtained in the undergraduate thesis of A. Scherlis.
} 


\subsection{Summary}

The results of this section can be summarized in the following classification of weight- 4 cluster functions on $E_{6} \cong \operatorname{Gr}(4,7)$ :

There are a total of 4193 irreducible weight-four cluster $\mathcal{A}$-functions

$\hookrightarrow 1155$ classical, 770 of which are physical

$\downarrow 3038$ non-classical, 126 of which are physical

$\hookrightarrow 2520 P B_{1}$ functions, 105 of which are physical

$\hookrightarrow 448 A_{2}$ functions; these are all of the cluster $\mathcal{X}$-functions

$\left\llcorner 195 P B_{0}\right.$ function, 14 of which are physical

$\left\llcorner 253 P B_{*}\right.$ functions

$\hookrightarrow 70$ other $P B_{*}$ functions

Again let us emphasize that these numbers count only irreducible functions, and that starting from the third line they moreover count functions modulo the classical function $\mathrm{Li}_{4}$ (i.e., the numbers refer to dimensions of subspaces of $B_{2} \wedge B_{2}$ ). When we say that a function is physical modulo additional terms, we mean that it is possible to choose the additional terms to render the function physical.

\subsection{The two-loop heptagon MHV amplitude}

The symbol of the two-loop seven-point MHV remainder function $R_{7}^{(2)}$ was computed in [25], and its cobracket was computed in [1], where it was observed to be a cluster $\mathcal{X}$-function living in $P B_{0}$. An analytic formula for $R_{7}^{(2)}$ was obtained in [26] and checked against the earlier numerical results of [27].

If we start from the hypothesis that $R_{7}^{(2)}$ should be a cluster $\mathcal{X}$-function living in $P B_{0}$, then we see from the above chart that there are only 14 physical functions with these properties. It was shown in [3] that only one linear combination of these has the dihedral symmetry required of the amplitude, is well-defined in the collinear limit, and satisfies the "last-entry" condition [25] required by supersymmetry.

In fact these constraints, while all true, are vastly stronger than necessary to pin down $R_{7}^{(2)}$ : in [12] it was found that the symbol of $R_{7}^{(2)}$ is the unique weight- 4 heptagon function (up to an overall multiplicative factor) which is well-defined in all $i+1 \| i$ collinear limits!

\subsection{The two-loop heptagon NMHV amplitude}

The symbol of the seven-point 2-loop NMHV ratio function $\mathcal{P}_{7, \mathrm{NMHV}}^{(2)}$ was first computed in [11]. It may be expressed as a linear combination of the 21 seven-point NMHV $R$ invariants (of which 15 are linearly independent), with coefficients that have uniform transcendentality weight 4 . Due to the linear relations between $R$-invariants there is some freedom in how to represent the amplitude (i.e., one can shift terms from one transcendental function to another by adding zero to the amplitude in various ways).

Despite this freedom, we find that it impossible to write the $B_{2} \wedge B_{2}$ cobracket of this amplitude in a Stasheff local manner, i.e. in terms of $\{x\}_{2} \wedge\{y\}_{2}$ for pairs $x, y$ having 
Poisson bracket $0, \pm 1$. The local terms having "good" Poisson brackets may be expressed (in one particular representation of the amplitude) as

$$
\delta_{22} \mathcal{P}_{7, \mathrm{NMHV}}^{(2)} \mid \text { "good" }=\left(f_{12} R_{12}+f_{13} R_{13}+f_{14} R_{14}\right)+\text { cyclic },
$$

where the quantities $f_{12}, f_{13}$ and $f_{13}$ are presented explicitly in the appendix, and $R_{i j}$ is the $R$-invariant whose arguments are 1234567 (in that order) but with $i$ and $j$ omitted this is the same as the notation used in [4]. Meanwhile the "bad" terms are given by:

$$
\delta_{22} \mathcal{P}_{7, \mathrm{NMHV}}^{(2)} \mid \text { "bad" }=\left(R_{25}-R_{26}+R_{37}-R_{47}\right) B_{1}+\text { cyclic }
$$

in terms of a single element $B_{1} \in B_{2} \wedge B_{2}$ (also given in the appendix) which is not expressible solely in terms of pairs having Poisson bracket zero or one.

In fact we can point our finger directly at the "offending" function corresponding to $B_{1}$ in the summary presented at the end of section 4.4. There we found that of the 126 non-classical weight-4 heptagon functions, 105 live in $P B_{1}$ while 14 live in $P B_{0}$, leaving $127-105-14=7$ unaccounted for. These other seven functions have $B_{2} \wedge B_{2}$ cobracket components given exactly by $B_{1}$ in its seven cyclic arrangements.

\section{Conclusion}

In this paper we have studied in detail the taxonomy of weight- 4 cluster functions on the cluster algebras relevant for 6- and seven-point amplitudes in planar SYM theory. In particular we have counted the numbers of linearly independent functions satisfying various mathematical constraints on their cobrackets, and the physical "first-entry" constraint which specifies the locations where amplitudes are permitted to have branch points on the principal sheet. These results are summarized in sections 3.4 and 4.4.

For $n=6$ the story is very simple: there is no non-classical weight- 4 generalized polylogarithm function which is consistent with the discrete symmetries of the MHV amplitude and whose $B_{2} \wedge B_{2}$ cobracket component is expressible in terms of pairs of cluster $\mathcal{X}$-coordinates having Poisson bracket 0 . This "explains" why the two-loop six-point MHV remainder function "must be" expressible in terms of classical polylogarithms [13].

Meanwhile, there are precisely 4 linearly independent non-classical functions which satisfy the first-entry condition and are Stasheff local (they have $B_{2} \wedge B_{2}$ cobracket components are expressible in terms of pairs of cluster $\mathcal{X}$-coordinates having Poisson bracket 1 ). These are precisely the (non-classical parts of the) 4 independent functions which appear in the two-loop six-point NMHV ratio function [4].

For $n=7$, as has already been observed in $[3,12]$, the cobracket (indeed, the whole symbol) of the two-loop MHV amplitude is uniquely determined by a simple list of mathematical and physical constraints. However the story for the two-loop NMHV ratio function is a little more complicated. We find that the cobracket of this amplitude is not expressible in a Stasheff local manner (that means, in terms of pairs having Poisson bracket $0, \pm 1$ ). It would be very interesting to learn if there is some other question one may ask about the cluster structure of this amplitude, to which a more affirmative answer may be given. We 
expect to be the case since it is known that there is a cluster structure at the level of the integrand (aspects of which have been explored in [28, 29]), of which some echo ought to remain for integrated amplitudes.

One of our results might be of more mathematical than physical interest. For both the $A_{3}$ and $E_{6}$ cluster algebras, we find that for any pair of $\mathcal{X}$-coordinates with Poisson bracket $\{\log x, \log y\}=1$, there exists a weight- 4 cluster $\mathcal{A}$-function (that is, an integrable symbol whose letters are drawn from the alphabet of cluster coordinates) whose $B_{2} \wedge B_{2}$ cobracket component is $\{x\}_{2} \wedge\{y\}_{2}$. It would be interesting to learn if there is a mathematical explanation for this fact, and whether it is valid for more general cluster algebras (in particular, for ones of infinite type). In contrast, pairs of $\mathcal{X}$-coordinates having Poisson bracket 0 are rarely integrable in this manner; the two-loop MHV amplitudes of planar SYM theory remarkably provide functions of this relatively rare type.

In the introduction we mentioned that in previous work including [3] it has been remarked that the mathematical and physical constraints on MHV amplitudes seem almost orthogonal. This is both good and bad. On the one hand it is good to discover a short list of simple criteria which uniquely, or almost uniquely, determine an amplitude of interest

- this is the core goal of the $S$-matrix program. On the other hand it is bad when there is no known formalism which simultaneously manifests both types of constraints. We do not yet know of any way, besides explicit enumeration, to actually identify and write down functions satisfying both the physical and mathematical we expect amplitudes to possess. Explicit results for higher loop planar SYM amplitudes remain, at least for the moment, difficult needles to find.

\section{Acknowledgments}

We have benefitted from stimulating discussions, correspondence, and collaboration with James Drummond, John Golden, Alexander Goncharov, Daniel Parker, Adam Scherlis, Cristian Vergu, and Anastasia Volovich. We are especially grateful to Lance Dixon for careful comments on the draft. MS is grateful to the CERN theory group for hospitality during the course of this work, which was supported by the US Department of Energy under contract DE-SC0010010 Task A.

\section{A Two-loop heptagon NMHV coproduct data}

In the first three subsections we list the Stasheff local contributions to the $B_{2} \wedge B_{2}$ cobracket component of the two-loop heptagon NMHV ratio function, in terms of the quantities $f_{12}$, $f_{13}$, and $f_{14}$ appearing in (4.5). Specifically, these contain all terms of the form $\{x\}_{2} \wedge\{y\}_{2}$ for pairs $x, y$ having Poisson bracket $0, \pm 1$. The additional "bad" contributions to the cobracket are shown in (4.6) and given explicitly in the fourth subsection. 


\section{A. $1 \quad f_{13}$}

This function is cyclically invariant and lives entirely in $P B_{1}$. We find

$$
\begin{aligned}
\delta_{22} f_{13}= & \frac{1}{7} \\
& \left(\left\{\frac{\langle 1367\rangle\langle 2347\rangle}{\langle 1237\rangle\langle 3467\rangle}\right\}_{2} \wedge\left\{\frac{\langle 1367\rangle\langle 2347\rangle\langle 4567\rangle}{\langle 1467\rangle\langle 2367\rangle\langle 3457\rangle}\right\}_{2}\right. \\
& -\left\{\frac{\langle 1247\rangle\langle 1256\rangle}{\langle 1245\rangle\langle 1267\rangle}\right\}_{2} \wedge\left\{\frac{\langle 1245\rangle\langle 1567\rangle}{\langle 1257\rangle\langle 1456\rangle}\right\}_{2} \\
& +\left\{\frac{\langle 1256\rangle\langle 2345\rangle}{\langle 1235\rangle\langle 2456\rangle}\right\}_{2} \wedge\left(\left\{\frac{\langle 1236\rangle\langle 1245\rangle}{\langle 1234\rangle\langle 1256\rangle}\right\}_{2}-\left\{\frac{\langle 1235\rangle\langle 1567\rangle\langle 2456\rangle}{\langle 1257\rangle\langle 1456\rangle\langle 2356\rangle}\right\}_{2}\right) \\
& +\left\{\frac{\langle 1247\rangle\langle 1345\rangle}{\langle 1234\rangle\langle 1457\rangle}\right\}_{2} \wedge\left(\left\{\frac{\langle 1345\rangle\langle 1467\rangle}{\langle 1347\rangle\langle 1456\rangle}\right\}_{2}-\left\{\frac{\langle 1245\rangle\langle 1467\rangle}{\langle 1247\rangle\langle 1456\rangle}\right\}_{2}\right) \\
& +\left(\left\{\frac{\langle 1247\rangle\langle 1345\rangle\langle 1567\rangle}{\langle 1257\rangle\langle 1347\rangle\langle 1456\rangle}\right\}_{2}-\left\{\frac{\langle 1247\rangle\langle 1256\rangle\langle 1345\rangle}{\langle 1234\rangle\langle 1257\rangle\langle 1456\rangle}\right\}_{2}\right) \\
& \wedge\left(\left\{-\frac{\langle 1267\rangle\langle 1345\rangle}{\langle 1(27)(34)(56)\rangle}\right\}_{2}+\left\{-\frac{\langle 1237\rangle\langle 1456\rangle}{\langle 1(27)(34)(56)\rangle}\right\}_{2}\right) \\
& \left.+\left(\left\{\frac{\langle 1247\rangle\langle 1256\rangle\langle 1346\rangle}{\langle 1234\rangle\langle 1267\rangle\langle 1456\rangle}\right\}_{2}-\left\{\frac{\langle 1237\rangle\langle 1345\rangle\langle 1567\rangle}{\langle 1257\rangle\langle 1347\rangle\langle 1356\rangle}\right\}_{2}\right) \wedge\left\{-\frac{\langle 1234\rangle\langle 1567\rangle}{\langle 1(27)(34)(56)\rangle}\right\}_{2}\right) \\
& +\operatorname{cyclic.}
\end{aligned}
$$

\section{A.2 $f_{12}$}

If we first define the quantity $X_{1}$ by

$$
\begin{aligned}
X_{1}= & \left\{\frac{\langle 1367\rangle\langle 2347\rangle}{\langle 1237\rangle\langle 3467\rangle}\right\}_{2} \wedge\left\{\frac{\langle 1267\rangle\langle 3467\rangle}{\langle 1467\rangle\langle 2367\rangle}\right\}_{2}+\left\{\frac{\langle 1467\rangle\langle 2347\rangle}{\langle 1247\rangle\langle 3467\rangle}\right\}_{2} \wedge\left\{\frac{\langle 1347\rangle\langle 4567\rangle}{\langle 1467\rangle\langle 3457\rangle}\right\}_{2} \\
& -\left\{\frac{\langle 1247\rangle\langle 1345\rangle}{\langle 1234\rangle\langle 1457\rangle}\right\}_{2} \wedge\left\{\frac{\langle 1245\rangle\langle 3457\rangle}{\langle 1457\rangle\langle 2345\rangle}\right\}_{2}-\left\{\frac{\langle 1457\rangle\langle 2347\rangle}{\langle 1247\rangle\langle 3457\rangle}\right\}_{2} \wedge\left\{\frac{\langle 1347\rangle\langle 4567\rangle}{\langle 1467\rangle\langle 3457\rangle}\right\}_{2} \\
& +\left\{\frac{\langle 1256\rangle\langle 2345\rangle}{\langle 1235\rangle\langle 2456\rangle}\right\}_{2} \wedge\left\{\frac{\langle 1236\rangle\langle 1245\rangle\langle 2567\rangle}{\langle 1235\rangle\langle 1267\rangle\langle 2456\rangle}\right\}_{2}-\left\{\frac{\langle 1267\rangle\langle 2356\rangle}{\langle 1236\rangle\langle 2567\rangle}\right\}_{2} \wedge\left\{\frac{\langle 1236\rangle\langle 2345\rangle\langle 2567\rangle}{\langle 1235\rangle\langle 2367\rangle\langle 2456\rangle}\right\}_{2} \\
& \left.+\left\{\frac{\langle 1234\rangle\langle 1467\rangle\langle 3457\rangle}{\langle 1247\rangle\langle 1345\rangle\langle 3467\rangle}\right\}_{2}-\left\{\frac{\langle 1245\rangle\langle 1467\rangle\langle 3457\rangle}{\langle 1247\rangle\langle 1345\rangle\langle 4567\rangle}\right\}_{2}\right) \wedge\left\{-\frac{\langle 1467\rangle\langle 2345\rangle}{\langle 4(12)(35)(67)\rangle}\right\}_{2} \\
& +\left\{\frac{\langle 1467\rangle\langle 2367\rangle\langle 2457\rangle}{\langle 1267\rangle\langle 2347\rangle\langle 4567\rangle}\right\}_{2} \wedge\left\{-\frac{\langle 1237\rangle\langle 4567\rangle}{\langle 7(16)(23)(45)\rangle}\right\}_{2} \\
& -\left\{\frac{\langle 1467\rangle\langle 2367\rangle\langle 3457\rangle}{\langle 1367\rangle\langle 2347\rangle\langle 4567\rangle}\right\}_{2} \wedge\left\{-\frac{\langle 1267\rangle\langle 3457\rangle}{\langle 7(16)(23)(45)\rangle}\right\}_{2} \\
& +2\left\{\frac{\langle 1245\rangle\langle 2467\rangle\langle 3457\rangle}{\langle 1247\rangle\langle 2345\rangle\langle 4567\rangle}\right\}_{2} \wedge\left\{-\frac{\langle 1234\rangle\langle 4567\rangle}{\langle 4(12)(35)(67)\rangle}\right\}_{2}
\end{aligned}
$$

and $X_{2}, \ldots, X_{7}$ by taking $i \rightarrow i+1$, then we find

$$
\begin{aligned}
\delta_{22} f_{12}= & \frac{1}{7}(3,-4,3,-4,3,-4,3) \cdot\left(X_{1}, X_{2}, X_{3}, X_{4}, X_{5}, X_{6}, X_{7}\right) \\
& +\left\{\frac{\langle 1237\rangle\langle 1246\rangle}{\langle 1234\rangle\langle 1267\rangle}\right\}_{2} \wedge\left(\left\{\frac{\langle 1246\rangle\langle 1345\rangle}{\langle 1234\rangle\langle 1456\rangle}\right\}_{2}+\left\{\frac{\langle 1234\rangle\langle 1467\rangle\langle 3456\rangle}{\langle 1246\rangle\langle 1345\rangle\langle 3467\rangle}\right\}_{2}+\left\{\frac{\langle 1467\rangle\langle 3456\rangle}{\langle 1346\rangle\langle 4567\rangle}\right\}_{2}\right. \\
& \left.+\left\{\frac{\langle 1246\rangle\langle 1345\rangle\langle 4567\rangle}{\langle 1245\rangle\langle 1467\rangle\langle 3456\rangle}\right\}_{2}\right)+\left\{\frac{\langle 1457\rangle\langle 3456\rangle}{\langle 1345\rangle\langle 4567\rangle}\right\}_{2} \wedge\left(\left\{\frac{\langle 1234\rangle\langle 1457\rangle}{\langle 1247\rangle\langle 1345\rangle}\right\}_{2}\right. \\
& +\left\{\frac{\langle 1234\rangle\langle 1267\rangle\langle 1457\rangle}{\langle 1237\rangle\langle 1245\rangle\langle 1467\rangle}\right\}_{2}+\left\{\frac{\langle 1237\rangle\langle 1467\rangle}{\langle 1267\rangle\langle 1347\rangle}\right\}_{2}+\left\{\frac{\langle 1237\rangle\langle 1345\rangle\langle 1467\rangle}{\langle 1234\rangle\langle 1367\rangle\langle 1457\rangle}\right\}_{2}
\end{aligned}
$$




$$
\begin{aligned}
& \left.+\left\{\frac{\langle 1257\rangle\langle 1456\rangle}{\langle 1245\rangle\langle 1567\rangle}\right\}_{2}\right)-\left\{\frac{\langle 1256\rangle\langle 2345\rangle}{\langle 1235\rangle\langle 2456\rangle}\right\}_{2} \wedge\left\{\frac{\langle 2567\rangle\langle 3456\rangle}{\langle 2356\rangle\langle 4567\rangle}\right\}_{2}+\left\{\frac{\langle 1267\rangle\langle 2356\rangle}{\langle 1236\rangle\langle 2567\rangle}\right\}_{2} \\
& \wedge\left\{\frac{\langle 1236\rangle\langle 2345\rangle\langle 3567\rangle}{\langle 1235\rangle\langle 2367\rangle\langle 3456\rangle}\right\}_{2}-\left\{\frac{\langle 1247\rangle\langle 1345\rangle}{\langle 1234\rangle\langle 1457\rangle}\right\}_{2} \wedge\left\{\frac{\langle 1247\rangle\langle 1567\rangle\langle 3457\rangle}{\langle 1257\rangle\langle 1347\rangle\langle 4567\rangle}\right\}_{2} \\
& +\left(\left\{\frac{\langle 1247\rangle\langle 1256\rangle\langle 1345\rangle}{\langle 1234\rangle\langle 1257\rangle\langle 1456\rangle}\right\}_{2}+\left\{\frac{\langle 1257\rangle\langle 1347\rangle\langle 1456\rangle}{\langle 1247\rangle\langle 1345\rangle\langle 1567\rangle}\right\}_{2}\right) \wedge\left\{-\frac{\langle 1247\rangle\langle 1567\rangle\langle 3456\rangle}{\langle 4567\rangle\langle 1(27)(34)(56)\rangle}\right\}_{2} \\
& +\left(\left\{\frac{\langle 1235\rangle\langle 2367\rangle\langle 2456\rangle}{\langle 1236\rangle\langle 2345\rangle\langle 2567\rangle}\right\}_{2}-\left\{\frac{\langle 1235\rangle\langle 1267\rangle\langle 2456\rangle}{\langle 1236\rangle\langle 1245\rangle\langle 2567\rangle}\right\}_{2}\right) \wedge\left\{-\frac{\langle 1236\rangle\langle 2345\rangle\langle 4567\rangle}{\langle 3456\rangle\langle 2(13)(45)(67)\rangle}\right\}_{2} \\
& +\left\{\frac{\langle 1467\rangle\langle 3457\rangle}{\langle 1347\rangle\langle 4567\rangle}\right\}_{2} \wedge\left(\left\{\frac{\langle 1237\rangle\langle 1467\rangle}{\langle 1267\rangle\langle 1347\rangle}\right\}_{2}-\left\{\frac{\langle 1267\rangle\langle 1347\rangle\langle 4567\rangle}{\langle 1247\rangle\langle 1567\rangle\langle 3467\rangle}\right\}_{2}\right)+\left\{\frac{\langle 2367\rangle\langle 3456\rangle}{\langle 2346\rangle\langle 3567\rangle}\right\}_{2} \\
& \wedge\left(\left\{\frac{\langle 1234\rangle\langle 2367\rangle}{\langle 1237\rangle\langle 2346\rangle}\right\}_{2}+\left\{\frac{\langle 1234\rangle\langle 2367\rangle\langle 3456\rangle}{\langle 1236\rangle\langle 2345\rangle\langle 3467\rangle}\right\}_{2}\right)+\frac{4}{7}\left(\left\{\frac{\langle 1257\rangle\langle 1456\rangle\langle 2356\rangle}{\langle 1235\rangle\langle 1567\rangle\langle 2456\rangle}\right\}_{2}\right. \\
& \wedge\left\{-\frac{\langle 1235\rangle\langle 4567\rangle}{\langle 5(17)(23)(46)\rangle}\right\}_{2}+\left\{\frac{\langle 1357\rangle\langle 1456\rangle\langle 2356\rangle}{\langle 1235\rangle\langle 1567\rangle\langle 3456\rangle}\right\}_{2} \wedge\left\{-\frac{\langle 1567\rangle\langle 2345\rangle}{\langle 5(17)(23)(46)\rangle}\right\}_{2} \\
& +\left\{\frac{\langle 1247\rangle\langle 1256\rangle\langle 1345\rangle}{\langle 1234\rangle\langle 1257\rangle\langle 1456\rangle}\right\}_{2} \wedge\left\{-\frac{\langle 1237\rangle\langle 1456\rangle}{\langle 1(27)(34)(56)\rangle}\right\}_{2}-\left\{\frac{\langle 1367\rangle\langle 2347\rangle\langle 3456\rangle}{\langle 1347\rangle\langle 2346\rangle\langle 3567\rangle}\right\}_{2} \\
& \left.\wedge\left\{-\frac{\langle 1367\rangle\langle 2345\rangle}{\langle 3(17)(24)(56)\rangle}\right\}_{2}\right)-\frac{3}{7}\left(\left\{\frac{\langle 1367\rangle\langle 1457\rangle\langle 2347\rangle}{\langle 1237\rangle\langle 1467\rangle\langle 3457\rangle}\right\}_{2} \wedge\left\{-\frac{\langle 1267\rangle\langle 3457\rangle}{\langle 7(16)(23)(45)\rangle}\right\}_{2}\right. \\
& +\left\{\frac{\langle 1236\rangle\langle 2567\rangle\langle 3467\rangle}{\langle 1267\rangle\langle 2346\rangle\langle 3567\rangle}\right\}_{2} \wedge\left\{-\frac{\langle 1567\rangle\langle 2346\rangle}{\langle 6(12)(34)(57)\rangle}\right\}_{2}+\left\{\frac{\langle 1235\rangle\langle 2367\rangle\langle 2456\rangle}{\langle 1236\rangle\langle 2345\rangle\langle 2567\rangle}\right\}_{2} \\
& \left.+\wedge\left\{-\frac{\langle 1234\rangle\langle 2567\rangle}{\langle 2(13)(45)(67)\rangle}\right\}_{2}+\left\{\frac{\langle 1245\rangle\langle 1467\rangle\langle 3457\rangle}{\langle 1247\rangle\langle 1345\rangle\langle 4567\rangle}\right\}_{2} \wedge\left\{-\frac{\langle 1247\rangle\langle 3456\rangle}{\langle 4(12)(35)(67)\rangle}\right\}_{2}\right) \\
& +\left(\left\{\frac{\langle 1235\rangle\langle 2367\rangle\langle 4567\rangle}{\langle 2567\rangle\langle 3(12)(45)(67)\rangle}\right\}_{2}-\left\{-\frac{\langle 1237\rangle\langle 2345\rangle\langle 4567\rangle}{\langle 3457\rangle\langle 2(13)(45)(67)\rangle}\right\}_{2}+\frac{4}{7}\left\{\frac{\langle 1235\rangle\langle 2367\rangle\langle 2457\rangle}{\langle 1237\rangle\langle 2345\rangle\langle 2567\rangle}\right\}_{2}\right) \\
& \wedge\left\{-\frac{\langle 1267\rangle\langle 2345\rangle}{\langle 2(13)(45)(67)\rangle}\right\}_{2}+\left(\left\{\frac{\langle 1237\rangle\langle 1345\rangle\langle 4567\rangle}{\langle 3457\rangle\langle 1(23)(45)(67)\rangle}\right\}_{2}-\left\{\frac{\langle 1236\rangle\langle 1345\rangle\langle 4567\rangle}{\langle 3456\rangle\langle 1(23)(45)(67)\rangle}\right\}_{2}\right. \\
& \left.-\left\{\frac{\langle 1237\rangle\langle 1456\rangle}{\langle 1(23)(45)(67)\rangle}\right\}_{2}\right) \wedge\left\{\frac{\langle 1267\rangle\langle 1345\rangle}{\langle 1(23)(45)(67)\rangle}\right\}_{2}+\left\{-\frac{\langle 1234\rangle\langle 1567\rangle}{\langle 1(27)(34)(56)\rangle}\right\}_{2} \\
& \wedge\left(\left\{-\frac{\langle 1237\rangle\langle 1567\rangle\langle 3456\rangle}{\langle 3567\rangle\langle 1(27)(34)(56)\rangle}\right\}_{2}-\left\{\frac{\langle 1257\rangle\langle 1347\rangle\langle 3456\rangle}{\langle 1345\rangle\langle 7(12)(34)(56)\rangle}\right\}_{2}+\frac{3}{7}\left\{\frac{\langle 1247\rangle\langle 1256\rangle\langle 1346\rangle}{\langle 1234\rangle\langle 1267\rangle\langle 1456\rangle}\right\}_{2}\right) \\
& +\left\{\frac{\langle 1237\rangle\langle 3456\rangle}{\langle 3(12)(45)(67)\rangle}\right\}_{2} \wedge\left(\left\{\frac{\langle 1267\rangle\langle 1345\rangle\langle 3467\rangle}{\langle 1467\rangle\langle 3(12)(45)(67)\rangle}\right\}_{2}+\left\{\frac{\langle 1234\rangle\langle 1367\rangle\langle 4567\rangle}{\langle 1467\rangle\langle 3(12)(45)(67)\rangle}\right\}_{2}\right. \\
& \left.-\left\{\frac{\langle 1234\rangle\langle 1267\rangle\langle 1345\rangle\langle 4567\rangle}{\langle 1245\rangle\langle 1467\rangle\langle 3(12)(45)(67)\rangle}\right\}_{2}\right)+\left(\left\{\frac{\langle 1234\rangle\langle 1267\rangle\langle 3457\rangle\langle 4567\rangle}{\langle 1247\rangle\langle 3467\rangle\langle 5(12)(34)(67)\rangle}\right\}_{2}\right. \\
& +\left\{-\frac{\langle 1234\rangle\langle 1267\rangle\langle 3456\rangle}{\langle 1236\rangle\langle 4(12)(35)(67)\rangle}\right\}_{2}-\left\{\frac{\langle 4567\rangle\langle 3(12)(45)(67)\rangle}{\langle 3467\rangle\langle 5(12)(34)(67)\rangle}\right\}_{2}-\frac{3}{7}\left\{\frac{\langle 1245\rangle\langle 2467\rangle\langle 3457\rangle}{\langle 1247\rangle\langle 2345\rangle\langle 4567\rangle}\right\}_{2} \\
& \left.-\left\{-\frac{\langle 1247\rangle\langle 3456\rangle}{\langle 4(12)(35)(67)\rangle}\right\}_{2}\right) \wedge\left\{-\frac{\langle 1234\rangle\langle 4567\rangle}{\langle 4(12)(35)(67)\rangle}\right\}_{2} \\
& +\left(\left\{\frac{\langle 1234\rangle\langle 1267\rangle\langle 3456\rangle\langle 3567\rangle}{\langle 1236\rangle\langle 3467\rangle\langle 5(12)(34)(67)\rangle}\right\}_{2}+\left\{-\frac{\langle 1234\rangle\langle 1267\rangle\langle 3567\rangle}{\langle 1237\rangle\langle 6(12)(34)(57)\rangle}\right\}_{2}\right. \\
& \left.+\left\{\frac{\langle 3467\rangle\langle 5(12)(34)(67)\rangle}{\langle 3456\rangle\langle 7(12)(34)(56)\rangle}\right\}_{2}-\frac{3}{7}\left\{\frac{\langle 1246\rangle\langle 2567\rangle\langle 3467\rangle}{\langle 1267\rangle\langle 2346\rangle\langle 4567\rangle}\right\}_{2}\right) \wedge\left\{-\frac{\langle 1267\rangle\langle 3456\rangle}{\langle 6(12)(34)(57)\rangle}\right\}_{2} \\
& +\left(\left\{-\frac{\langle 1267\rangle\langle 3457\rangle}{\langle 7(16)(23)(45)\rangle}\right\}_{2}+\frac{4}{7}\left\{\frac{\langle 1367\rangle\langle 1457\rangle\langle 2357\rangle}{\langle 1237\rangle\langle 1567\rangle\langle 3457\rangle}\right\}_{2}-\left\{\frac{\langle 1467\rangle\langle 2367\rangle\langle 3457\rangle}{\langle 1367\rangle\langle 2347\rangle\langle 4567\rangle}\right\}_{2}\right)
\end{aligned}
$$




$$
\begin{aligned}
& \wedge\left\{-\frac{\langle 1237\rangle\langle 4567\rangle}{\langle 7(16)(23)(45)\rangle}\right\}_{2}+\left(\left\{-\frac{\langle 1234\rangle\langle 3567\rangle}{\langle 3(17)(24)(56)\rangle}\right\}_{2}-\frac{3}{7}\left\{\frac{\langle 1347\rangle\langle 1356\rangle\langle 2346\rangle}{\langle 1234\rangle\langle 1367\rangle\langle 3456\rangle}\right\}_{2}\right. \\
& \left.+\left\{\frac{\langle 1367\rangle\langle 2347\rangle\langle 3456\rangle}{\langle 1347\rangle\langle 2346\rangle\langle 3567\rangle}\right\}_{2}\right) \wedge\left\{-\frac{\langle 1237\rangle\langle 3456\rangle}{\langle 3(17)(24)(56)\rangle}\right\}_{2} .
\end{aligned}
$$

\section{A.3 $f_{14}$}

This function lives entirely in $P B_{1}$. If we first define the quantity

$$
\begin{aligned}
Y= & \left\{\frac{\langle 2347\rangle\langle 2356\rangle}{\langle 2345\rangle\langle 2367\rangle}\right\}_{2} \wedge\left\{\frac{\langle 2346\rangle\langle 3567\rangle}{\langle 2367\rangle\langle 3456\rangle}\right\}_{2}+\left\{\frac{\langle 1367\rangle\langle 2347\rangle}{\langle 1237\rangle\langle 3467\rangle}\right\}_{2} \wedge\left\{\frac{\langle 2347\rangle\langle 3567\rangle}{\langle 2367\rangle\langle 3457\rangle}\right\}_{2} \\
& +\left\{\frac{\langle 1257\rangle\langle 1456\rangle}{\langle 1245\rangle\langle 1567\rangle}\right\}_{2} \wedge\left\{\frac{\langle 1257\rangle\langle 1456\rangle\langle 2345\rangle}{\langle 1235\rangle\langle 1457\rangle\langle 2456\rangle}\right\}_{2}-\left\{\frac{\langle 1367\rangle\langle 1457\rangle\langle 2347\rangle}{\langle 1237\rangle\langle 1467\rangle\langle 3457\rangle}\right\}_{2} \wedge\left\{\frac{\langle 1347\rangle\langle 4567\rangle}{\langle 1467\rangle\langle 3457\rangle}\right\}_{2} \\
& -\left\{\frac{\langle 1237\rangle\langle 2356\rangle}{\langle 1235\rangle\langle 2367\rangle}\right\}_{2} \wedge\left\{\frac{\langle 1236\rangle\langle 2567\rangle}{\langle 1267\rangle\langle 2356\rangle}\right\}_{2}-\left\{\frac{\langle 1256\rangle\langle 2345\rangle}{\langle 1235\rangle\langle 2456\rangle}\right\}_{2} \wedge\left\{\frac{\langle 1235\rangle\langle 2567\rangle}{\langle 1257\rangle\langle 2356\rangle}\right\}_{2} \\
& +\left\{\frac{\langle 1257\rangle\langle 1456\rangle\langle 2345\rangle}{\langle 1235\rangle\langle 1457\rangle\langle 2456\rangle}\right\}_{2} \wedge\left\{-\frac{\langle 1235\rangle\langle 4567\rangle}{\langle 5(17)(23)(46)\rangle}\right\}_{2} \\
& -\left\{\frac{\langle 1257\rangle\langle 1456\rangle\langle 2356\rangle}{\langle 1235\rangle\langle 1567\rangle\langle 2456\rangle}\right\}_{2} \wedge\left\{-\frac{\langle 1235\rangle\langle 4567\rangle}{\langle 5(17)(23)(46)\rangle}\right\}_{2} \\
& +\left\{\frac{\langle 1235\rangle\langle 2367\rangle\langle 2457\rangle}{\langle 1237\rangle\langle 2345\rangle\langle 2567\rangle}\right\}_{2} \wedge\left\{-\frac{\langle 1267\rangle\langle 2345\rangle}{\langle 2(13)(45)(67)\rangle}\right\}_{2} \\
& -\left\{\frac{\langle 1367\rangle\langle 1457\rangle\langle 2347\rangle}{\langle 1237\rangle\langle 1467\rangle\langle 3457\rangle}\right\}_{2} \wedge\left\{-\frac{\langle 1567\rangle\langle 2347\rangle}{\langle 7(16)(23)(45)\rangle}\right\}_{2} \\
& +\left\{\frac{\langle 1357\rangle\langle 2347\rangle\langle 2356\rangle}{\langle 1237\rangle\langle 2345\rangle\langle 3567\rangle}\right\}_{2} \wedge\left\{-\frac{\langle 1237\rangle\langle 3456\rangle}{\langle 3(17)(24)(56)\rangle}\right\}_{2} \\
& -\left\{\frac{\langle 1467\rangle\langle 2367\rangle\langle 3457\rangle}{\langle 1367\rangle\langle 2347\rangle\langle 4567\rangle}\right\}_{2} \wedge\left\{-\frac{\langle 1567\rangle\langle 2347\rangle}{\langle 7(16)(23)(45)\rangle}\right\}_{2}
\end{aligned}
$$

then we find

$$
\begin{aligned}
\delta_{22} f_{14}= & \frac{2}{7}(Y+\text { cyclic })-2 Y+\left(\left\{\frac{\langle 1257\rangle\langle 1456\rangle}{\langle 1245\rangle\langle 1567\rangle}\right\}_{2}-\left\{\frac{\langle 1267\rangle\langle 2356\rangle}{\langle 1236\rangle\langle 2567\rangle}\right\}_{2}-\left\{\frac{\langle 1257\rangle\langle 2456\rangle}{\langle 1245\rangle\langle 2567\rangle}\right\}_{2}\right. \\
& \left.+\left\{\frac{\langle 1235\rangle\langle 1267\rangle\langle 2456\rangle}{\langle 1236\rangle\langle 1245\rangle\langle 2567\rangle}\right\}_{2}\right) \wedge\left\{\frac{\langle 1256\rangle\langle 2345\rangle}{\langle 1235\rangle\langle 2456\rangle}\right\}_{2}+\left\{\frac{\langle 1235\rangle\langle 2367\rangle\langle 2456\rangle}{\langle 1236\rangle\langle 2345\rangle\langle 2567\rangle}\right\}_{2} \\
& \wedge\left(\left\{\frac{\langle 1267\rangle\langle 2356\rangle}{\langle 1236\rangle\langle 2567\rangle}\right\}_{2}-\left\{-\frac{\langle 1267\rangle\langle 2345\rangle}{\langle 2(13)(45)(67)\rangle}\right\}_{2}\right)+\left(\left\{\frac{\langle 1235\rangle\langle 2367\rangle\langle 2456\rangle}{\langle 1236\rangle\langle 2345\rangle\langle 2567\rangle}\right\}_{2}\right. \\
& \left.+\left\{\frac{\langle 1236\rangle\langle 1245\rangle\langle 2567\rangle}{\langle 1235\rangle\langle 1267\rangle\langle 2456\rangle}\right\}_{2}\right) \wedge\left\{-\frac{\langle 1237\rangle\langle 2456\rangle}{\langle 2(13)(45)(67)\rangle}\right\}_{2}-\left\{\frac{\langle 1367\rangle\langle 1457\rangle\langle 2357\rangle}{\langle 1237\rangle\langle 1567\rangle\langle 3457\rangle}\right\}_{2} \\
& \wedge\left\{-\frac{\langle 1237\rangle\langle 4567\rangle}{\langle 7(16)(23)(45)\rangle}\right\}_{2}-\left\{\frac{\langle 1367\rangle\langle 2347\rangle\langle 3456\rangle}{\langle 1347\rangle\langle 2346\rangle\langle 3567\rangle}\right\}_{2} \wedge\left\{-\frac{\langle 1237\rangle\langle 3456\rangle}{\langle 3(17)(24)(56)\rangle}\right\}_{2} \\
& +\left(\left\{\frac{\langle 1367\rangle\langle 2347\rangle\langle 2356\rangle}{\langle 1237\rangle\langle 2346\rangle\langle 3567\rangle}\right\}_{2}+\left\{\frac{\langle 1347\rangle\langle 2346\rangle\langle 3567\rangle}{\langle 1367\rangle\langle 2347\rangle\langle 3456\rangle}\right\}_{2}\right) \wedge\left\{-\frac{\langle 1367\rangle\langle 2345\rangle}{\langle 3(17)(24)(56)\rangle}\right\}_{2} \\
& -\left\{\frac{\langle 2346\rangle\langle 3567\rangle}{\langle 2367\rangle\langle 3456\rangle}\right\}_{2} \wedge\left\{\frac{\langle 1237\rangle\langle 2346\rangle\langle 3567\rangle}{\langle 1367\rangle\langle 2347\rangle\langle 2356\rangle}\right\}_{2}-\left(\left\{\frac{\langle 1457\rangle\langle 2456\rangle}{\langle 1245\rangle\langle 4567\rangle}\right\}_{2}\right. \\
& \left.+\left\{\frac{\langle 1567\rangle\langle 2456\rangle}{\langle 1256\rangle\langle 4567\rangle}\right\}_{2}\right) \wedge\left\{\frac{\langle 1257\rangle\langle 1456\rangle}{\langle 1245\rangle\langle 1567\rangle}\right\}_{2}-\left\{\frac{\langle 1457\rangle\langle 2357\rangle\langle 2456\rangle}{\langle 1257\rangle\langle 2345\rangle\langle 4567\rangle}\right\}_{2} \wedge\left\{-\frac{\langle 1567\rangle\langle 2345\rangle}{\langle 5(17)(23)(46)\rangle}\right\}_{2} \\
& -\left(\left\{\frac{\langle 1567\rangle\langle 2357\rangle\langle 2456\rangle}{\langle 1257\rangle\langle 2356\rangle\langle 4567\rangle}\right\}_{2}+\left\{\frac{\langle 1567\rangle\langle 2357\rangle\langle 3456\rangle}{\langle 1357\rangle\langle 2356\rangle\langle 4567\rangle}\right\}_{2}\right) \wedge\left\{-\frac{\langle 1567\rangle\langle 2345\rangle}{\langle 5(17)(23)(46)\rangle}\right\}_{2} \\
& +\left(\left\{\frac{\langle 1347\rangle\langle 1567\rangle}{\langle 1367\rangle\langle 1457\rangle}\right\}_{2}+\left\{\frac{\langle 1567\rangle\langle 3467\rangle}{\langle 1367\rangle\langle 4567\rangle}\right\}_{2}\right) \wedge\left\{\frac{\langle 1467\rangle\langle 3457\rangle}{\langle 1347\rangle\langle 4567\rangle}\right\}_{2}-\left(\left\{\frac{\langle 1347\rangle\langle 2346\rangle\langle 3567\rangle}{\langle 1367\rangle\langle 2347\rangle\langle 3456\rangle}\right\}_{2}\right.
\end{aligned}
$$




$$
\begin{aligned}
& \left.-\left\{\frac{\langle 2346\rangle\langle 3567\rangle}{\langle 2367\rangle\langle 3456\rangle}\right\}_{2}+\left\{\frac{\langle 1347\rangle\langle 3567\rangle}{\langle 1367\rangle\langle 3457\rangle}\right\}_{2}-\left\{\frac{\langle 1347\rangle\langle 4567\rangle}{\langle 1467\rangle\langle 3457\rangle}\right\}_{2}\right) \wedge\left\{\frac{\langle 1237\rangle\langle 3467\rangle}{\langle 1367\rangle\langle 2347\rangle}\right\}_{2} \\
& +\left(\left\{\frac{\langle 1237\rangle\langle 1467\rangle\langle 3457\rangle}{\langle 1367\rangle\langle 1457\rangle\langle 2347\rangle}\right\}_{2}+\left\{\frac{\langle 1567\rangle\langle 2367\rangle\langle 2457\rangle}{\langle 1267\rangle\langle 2357\rangle\langle 4567\rangle}\right\}_{2}+\left\{\frac{\langle 1567\rangle\langle 2367\rangle\langle 3457\rangle}{\langle 1367\rangle\langle 2357\rangle\langle 4567\rangle}\right\}_{2}\right. \\
& \left.+\left\{\frac{\langle 1367\rangle\langle 2347\rangle\langle 4567\rangle}{\langle 1467\rangle\langle 2367\rangle\langle 3457\rangle}\right\}_{2}\right) \wedge\left\{-\frac{\langle 1237\rangle\langle 4567\rangle}{\langle 7(16)(23)(45)\rangle}\right\}_{2} .
\end{aligned}
$$

\section{A.4 $B_{1}$}

Here we display the non-Stasheff local contributions to the $B_{2} \wedge B_{2}$ coproduct component of the two-loop seven-point NMHV ratio function (4.6). Exceptionally in this formula we make use of the cross-ratios $a_{i j}$ defined in equation (2.1) of [12]. We find that

$$
\begin{aligned}
B_{1}= & \left(a_{12} \wedge a_{16}\right) \wedge\left(a_{12} \wedge a_{61}\right)+\left(a_{12} \wedge a_{16}\right) \wedge\left(a_{17} \wedge a_{61}\right)-\left(a_{12} \wedge a_{23}\right) \wedge\left(a_{12} \wedge a_{61}\right) \\
& -\left(a_{12} \wedge a_{23}\right) \wedge\left(a_{17} \wedge a_{61}\right)-\left(a_{12} \wedge a_{32}\right) \wedge\left(a_{12} \wedge a_{61}\right)-\left(a_{12} \wedge a_{32}\right) \wedge\left(a_{17} \wedge a_{61}\right) \\
& -\left(a_{12} \wedge a_{61}\right) \wedge\left(a_{13} \wedge a_{16}\right)+\left(a_{12} \wedge a_{61}\right) \wedge\left(a_{13} \wedge a_{23}\right)+\left(a_{12} \wedge a_{61}\right) \wedge\left(a_{13} \wedge a_{32}\right) \\
& -\left(a_{12} \wedge a_{61}\right) \wedge\left(a_{16} \wedge a_{23}\right)-\left(a_{12} \wedge a_{61}\right) \wedge\left(a_{16} \wedge a_{32}\right)+\left(a_{13} \wedge a_{16}\right) \wedge\left(a_{17} \wedge a_{61}\right) \\
& -\left(a_{13} \wedge a_{23}\right) \wedge\left(a_{17} \wedge a_{61}\right)-\left(a_{13} \wedge a_{32}\right) \wedge\left(a_{17} \wedge a_{61}\right)+\left(a_{16} \wedge a_{23}\right) \wedge\left(a_{17} \wedge a_{61}\right) \\
& +\left(a_{16} \wedge a_{32}\right) \wedge\left(a_{17} \wedge a_{61}\right)
\end{aligned}
$$

where we follow the slight abuse of notation explained in [15] of writing $B_{1}$ not explicitly as an element of $B_{2} \wedge B_{2}$, but rather by writing the result of the iterated coproduct acting on $B_{1}$ according to $\{a\}_{2} \wedge\{b\}_{2} \mapsto(a \wedge(1+a)) \wedge(b \wedge(1+b))$ and then expanding all multiplicative terms out using the usual symbol rules. In other words, the above formula represents the symbol of the function $B_{1}$ antisymmetrized according to $a \otimes b \otimes c \otimes d \mapsto(a \wedge b) \wedge(c \wedge d)$.

Open Access. This article is distributed under the terms of the Creative Commons Attribution License (CC-BY 4.0), which permits any use, distribution and reproduction in any medium, provided the original author(s) and source are credited.

\section{References}

[1] J. Golden, A.B. Goncharov, M. Spradlin, C. Vergu and A. Volovich, Motivic amplitudes and cluster coordinates, JHEP 01 (2014) 091 [arXiv: 1305.1617] [INSPIRE].

[2] A.B. Goncharov, Galois symmetries of fundamental groupoids and noncommutative geometry, Duke Math. J. 128 (2005) 209 [math/0208144] [INSPIRE].

[3] J. Golden and M. Spradlin, A cluster bootstrap for two-loop MHV amplitudes, JHEP 02 (2015) 002 [arXiv: 1411.3289] [INSPIRE].

[4] L.J. Dixon, J.M. Drummond and J.M. Henn, Analytic result for the two-loop six-point NMHV amplitude in $N=4$ super Yang-Mills theory, JHEP 01 (2012) 024 [arXiv:1111.1704] [INSPIRE].

[5] L.J. Dixon, J.M. Drummond and J.M. Henn, Bootstrapping the three-loop hexagon, JHEP 11 (2011) 023 [arXiv:1108.4461] [INSPIRE].

[6] L.J. Dixon, J.M. Drummond, M. von Hippel and J. Pennington, Hexagon functions and the three-loop remainder function, JHEP 12 (2013) 049 [arXiv:1308.2276] [INSPIRE]. 
[7] L.J. Dixon, J.M. Drummond, C. Duhr and J. Pennington, The four-loop remainder function and multi-Regge behavior at NNLLA in planar $N=4$ super-Yang-Mills theory, JHEP 06 (2014) 116 [arXiv: 1402.3300] [INSPIRE].

[8] L.J. Dixon, J.M. Drummond, C. Duhr, M. von Hippel and J. Pennington, Bootstrapping six-gluon scattering in planar $N=4$ super-Yang-Mills theory, PoS(LL2014) 077 [arXiv: 1407.4724] [INSPIRE].

[9] L.J. Dixon and M. von Hippel, Bootstrapping an NMHV amplitude through three loops, JHEP 10 (2014) 065 [arXiv: 1408.1505] [INSPIRE].

[10] L.J. Dixon, M. von Hippel and A.J. McLeod, The four-loop six-gluon NMHV ratio function, JHEP 01 (2016) 053 [arXiv: 1509.08127] [INSPIRE].

[11] S. Caron-Huot and S. He, Jumpstarting the all-loop S-matrix of planar $N=4$ super Yang-Mills, JHEP 07 (2012) 174 [arXiv:1112.1060] [INSPIRE].

[12] J.M. Drummond, G. Papathanasiou and M. Spradlin, A symbol of uniqueness: the cluster bootstrap for the 3-loop MHV heptagon, JHEP 03 (2015) 072 [arXiv:1412.3763] [INSPIRE].

[13] A.B. Goncharov, M. Spradlin, C. Vergu and A. Volovich, Classical polylogarithms for amplitudes and Wilson loops, Phys. Rev. Lett. 105 (2010) 151605 [arXiv:1006.5703] [INSPIRE].

[14] C. Vergu, unpublished.

[15] J. Golden, M.F. Paulos, M. Spradlin and A. Volovich, Cluster polylogarithms for scattering amplitudes, J. Phys. A 47 (2014) 474005 [arXiv:1401.6446] [InSPIRE].

[16] D. Gaiotto, J. Maldacena, A. Sever and P. Vieira, Pulling the straps of polygons, JHEP 12 (2011) 011 [arXiv:1102.0062] [InSPIRE].

[17] A. Hodges, Eliminating spurious poles from gauge-theoretic amplitudes, JHEP 05 (2013) 135 [arXiv: 0905.1473] [INSPIRE].

[18] M. Gekhtman, M. Shapiro and A. Vainshtein, Cluster algebras and Poisson geometry, Mosc. Math. J. 3 (2003) 899 [math/0208033].

[19] J.S. Scott, Grassmannians and cluster algebras, Proc. Lond. Math. Soc. 92 (2006) 345.

[20] V.V. Fock and A.B. Goncharov, Cluster ensembles, quantization and the dilogarithm, Ann. Sci. Éc. Norm. Supér. 42 (2009) 865 [math/0311245] [INSPIRE].

[21] D. Parker, A. Scherlis, M. Spradlin and A. Volovich, Hedgehog bases for $A_{n}$ cluster polylogarithms and an application to six-point amplitudes, JHEP 11 (2015) 136 [arXiv: 1507.01950] [INSPIRE].

[22] F.C.S. Brown, Multiple zeta values and periods of moduli space $M_{0, n}$, Annales Sci. Ecole Norm. Sup. 42 (2009) 371 [math/0606419] [INSPIRE].

[23] S. Benvenuti, B. Feng, A. Hanany and Y.-H. He, Counting BPS operators in gauge theories: quivers, syzygies and plethystics, JHEP 11 (2007) 050 [hep-th/0608050] [INSPIRE].

[24] A.B. Goncharov, Polylogarithms and motivic Galois group, Proceedings of the Symposium on Pure Mathematics 55, American Mathematical Society, Providence U.S.A. (1994).

[25] S. Caron-Huot, Superconformal symmetry and two-loop amplitudes in planar $N=4$ super Yang-Mills, JHEP 12 (2011) 066 [arXiv:1105.5606] [INSPIRE]. 
[26] J. Golden and M. Spradlin, An analytic result for the two-loop seven-point MHV amplitude in $\mathcal{N}=4$ SYM, JHEP 08 (2014) 154 [arXiv:1406.2055] [INSPIRE].

[27] C. Anastasiou, A. Brandhuber, P. Heslop, V.V. Khoze, B. Spence and G. Travaglini, Two-loop polygon Wilson loops in $N=4$ SYM, JHEP 05 (2009) 115 [arXiv:0902.2245] [INSPIRE].

[28] N. Arkani-Hamed et al., Scattering amplitudes and the positive Grassmannian, arXiv: 1212.5605

[29] M.F. Paulos and B.U.W. Schwab, Cluster algebras and the positive Grassmannian, JHEP 10 (2014) 31 [arXiv:1406.7273] [INSPIRE]. 